\title{
Correlations in interacting systems with a network topology
}

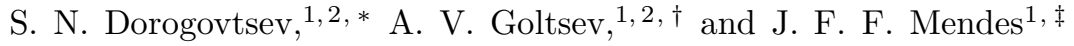 \\ ${ }^{1}$ Departamento de Física da Universidade de Aveiro, 3810-193 Aveiro, Portugal \\ ${ }^{2}$ A. F. Ioffe Physico-Technical Institute, 194021 St. Petersburg, Russia
}

(Dated:)

\begin{abstract}
We study pair correlations in cooperative systems placed on complex networks. We show that usually in these systems, the correlations between two interacting objects (e.g., spins), separated by a distance $\ell$, decay, on average, faster than $1 /\left(\ell z_{\ell}\right)$. Here $z_{\ell}$ is the mean number of the $\ell$ th nearest neighbors of a vertex in a network. This behavior, in particular, leads to a dramatic weakening of correlations between second and more distant neighbors on networks with fat-tailed degree distributions, which have a divergent number $z_{2}$ in the infinite network limit. In this case, only the pair correlations between the nearest neighbors are observable. We obtain the pair correlation function of the Ising model on a complex network and also derive our results in the framework of a phenomenological approach.
\end{abstract}

PACS numbers: 05.10.-a, 05-40.-a, 05-50.+q, 87.18.Sn

\section{INTRODUCTION.}

The generic features of real-world networks (the Internet, the WWW, biological, social and economical networks and many others) are a complex organization of their connections and the small-world phenomenon [1, 2, 3, 4, [5, 6, 7, 8]. In the networks with the smallworld effect, a mean intervertex distance grows with a total number of vertices, $N$, slower than any power of $N$, e.g., grows as $\ln N$. Concerning economical and social networks, the small-world property is often considered as an evidence for growing close interrelations and globalization.

Many of the real-world networks are formed by interacting objects and demonstrate complicated dynamics. The dependence of the correlations between interacting objects in systems with a network topology on time and distance provides a useful information about the network dynamics. In the present paper we discuss general properties of the correlations between a pair of interacting objects on a complex network. Recall that in interacting systems on lattices, with a few exceptions, pair correlations decrease exponentially with distance apart from a critical point, where the decrease is power-law. From a naive point of view, one might expect that the smallworld property of a network would enhance pair correlations between distant objects in comparison to lattices. However, it is not the case. We demonstrate that correlations between $\ell$-th nearest neighbors, on average, decay with $\ell$ as $1 /\left(\ell z_{\ell}\right)$ or faster. In networks, where the mean intervertex separation $\bar{\ell}(N) \sim \ln N$, this corresponds to the exponential decay of correlations with $\ell$ (even at the critical point). However, in networks with a divergent mean number of the second nearest neighbors

\footnotetext{
*Electronic address: sdorogov@fis.ua.pt

†Electronic address: goltsev@fis.ua.pt

${ }^{\ddagger}$ Electronic address: jfmendes@fis.ua.pt
}

[where $\bar{\ell}(N)$ grows slower than $\ln N$ ], we observe a dramatic weakening of pair correlations between the second and more distant nearest neighbors. In these networks, only the nearest neighbors are strongly correlated, while the correlations between more distant vertices are suppressed and approach zero in the infinite network limit.

In Section ஹw we consider pair correlations in an interacting system defined on the top of a complex network in the framework of a phenomenological approach. This approach allows us to understand general properties of pair correlations irrespective of details of an interacting system and the nature of interactions. In Section $[11$ we support these results by calculations of the static pair correlation function of the Ising model on a complex network (more precisely, the configuration model of a network $\underline{9}])$.

\section{PHENOMENOLOGICAL APPROACH}

Let a quantity $X_{i}(t)$ describe a dynamic process on a network, where indices $i$ label vertices, and $t$ is time. $x_{i}(t) \equiv X_{i}(t)-\left\langle X_{i}\right\rangle$ describes fluctuations around the average value $\left\langle X_{i}\right\rangle$. If the system under consideration is in an equilibrium state, then pair correlations between two arbitrary vertices $i$ and $j$ may be characterized by the following correlation function:

$$
\begin{aligned}
G_{i j}\left(t_{1}, t_{2}\right) & =t_{0}^{-1} \int_{0}^{t_{0}} x_{i}\left(t+t_{1}\right) x_{j}\left(t+t_{2}\right) d t \\
& \equiv\left\langle x_{i}\left(t_{1}\right) x_{j}\left(t_{2}\right)\right\rangle .
\end{aligned}
$$

In the present section the brackets $\langle\ldots\rangle$ mean the average over the observation time $t_{0}$ which must be much larger than the maximum relaxation time in the system under consideration. In the equilibrium state we have $G_{i j}\left(t_{1}, t_{2}\right)=G_{i j}\left(t_{1}-t_{2}\right)$ in the limit $t_{0} \rightarrow \infty$. Assuming the Hamiltonian dynamics, one can introduce a general- 
ized field $H_{i}(t)$ conjugated with $X_{i}(t)$. The function

$$
\begin{aligned}
\chi_{i j}\left(t_{1}-t_{2}\right) & =t_{0}^{-1} \int_{0}^{t_{0}} \partial x_{i}\left(t+t_{1}\right) / \partial H_{j}\left(t+t_{2}\right) d t \\
& \equiv\left\langle\partial x_{i}\left(t_{1}\right) / \partial H_{j}\left(t_{2}\right)\right\rangle
\end{aligned}
$$

is a generalized non-local susceptibility which characterizes the averaged response of $x_{i}\left(t_{1}\right)$ at time $t_{1}$ on a field applied at a vertex $j$ at time $t_{2}$. There is a simple relationship between $G_{i j}(t)$ and $\chi_{i j}(t)$ :

$$
G_{i j}(t) \propto \chi_{i j}(t)
$$

where coefficient of the proportionality is unimportant for our purpose.

In general case, the total susceptibility $\chi(t)$ of a system (per vertex) is finite in the limit $N \rightarrow \infty$ where $N$ is the number of vertices in a network:

$$
\chi\left(t_{1}-t_{2}\right)=\left.\frac{1}{N} \sum_{i=1}^{N}\left\langle\frac{\partial x_{i}\left(t_{1}\right)}{\partial H_{j}\left(t_{2}\right)}\right\rangle\right|_{H_{1}=H_{2}=\ldots=H}=O(1) .
$$

Here, a statement $A=O(1)$ means that a quantity $A$ is finite in the limit $N \rightarrow \infty$. The susceptibility $\chi(t)$ may diverge only at a critical point. We rewrite Eq. (4) as follows:

$$
\chi(t)=\frac{1}{N} \sum_{i}\left(\chi_{i i}(t)+\sum_{j: \ell_{i j}=1} \chi_{i j}(t)+\sum_{j: \ell_{i j}=2} \chi_{i j}(t)+\ldots\right),
$$

where the first sum in the parentheses is a sum over the nearest neighbors of a vertex $i$ being at the distance $\ell_{i j}=1$. The second sum is over the second nearest neighbors, $\ell_{i j}=2$, and so on. One can introduce the average value $\chi(t, \ell)$, that is the average value of the susceptibility $\chi_{i j}(t)$ at $\ell_{i j}=\ell$ over all possible network configurations. In the large network limit, this quantity coincides with the following expression calculated for a single network:

$$
\chi(t, \ell)=\frac{\sum_{i, j: \ell_{i j}=\ell} \chi_{i j}(t)}{\sum_{i, j: \ell_{i j}=\ell} 1}=\frac{\sum_{i, j: \ell_{i j}=\ell} \chi_{i j}(t)}{N z_{\ell}},
$$

where the sums are over all pairs of vertices at the intervertex distance $\ell, z_{\ell}=N^{-1} \sum_{i, j: \ell_{i j}=\ell} 1$ is the mean number of $\ell$-th nearest neighbors. Consequently,

$$
\chi(t)=\sum_{\ell} z_{\ell} \chi(t, \ell)
$$

where $z_{0}=1$ and $\chi(t, 0)$ is the average local susceptibility. Let us first assume that all $\chi(t, \ell) \geq 0$. In order to satisfy the condition $\chi(t)=O(1)$, the terms of the sum in Eq. (7) must decay with $\ell$ faster than $1 / \ell$. This leads to the following restriction:

$$
\chi(t, \ell)<O\left(\frac{1}{\ell z_{\ell}}\right)
$$

It is important that here $z_{\ell}$ is a function of the network size $N$, so formula (8) shows how the nonlocal susceptibility (and spacial correlations) vary with $N$.

If the nonlocal susceptibilities $\chi_{i j}(t)$ are of varying sign, formula (8) is not applicable. In this case, for the root-mean-square with averaging over all pairs of vertices $i, j$ with a given $\ell_{i j}=\ell$, we arrive at

$$
\left[\overline{\chi_{i j}^{2}\left(t, \ell_{i j}=\ell\right)}\right]^{1 / 2}<O\left(z_{\ell}^{-1 / 2}\right) .
$$

In accordance to Eq. (3), the $\ell$ dependence of the average correlation function is the same as for the average susceptibility, i.e., it is described by formulas (8) or (9).

In sparse networks, the mean number of the nearest neighbors (the mean degree) $z_{1} \equiv \bar{k}=\sum k P(k)$ is finite. In general, in networks, where the mean intervertex distance $\bar{\ell}(N) \sim N$, the mean numbers of $\ell$-th nearest neighbors grow exponentially with $\ell$. In particular, in uncorrelated networks (without degree-degree correlations between nearest neighbor vertices), $z_{\ell}=z_{1}\left(z_{2} / z_{1}\right)^{\ell-1}$, where $z_{2}=\sum k(k-1) P(k)$ [10]. In this case, even at the critical point of a cooperative model, the nonlocal susceptibility and pair correlations decrease exponentially rapidly with $\ell$.

The effect is even more dramatic, if the degree distribution is fat-tailed, and its second moment diverges as $N \rightarrow \infty$. In scale-free networks, this takes place if the $\gamma$ exponent of the degree distribution $P(k) \sim k^{-\gamma}$ is equal or below 3 . In this case, already the mean number $z_{2}$ of the second nearest neighbors of a vertex diverges as $N \rightarrow \infty$. Consequently, according to Eq. (8) or Eq. (9), in the limit of a large network, pair correlations between the second (and further) nearest neighbors vanish, and only pair correlations between the nearest neighbors are observable.

\section{CORRELATIONS IN THE ISING MODEL}

The simplicity of the Ising model and the fact that many microscopic models may be mapped to this model make the Ising model to be very attractive. Recent investigations have revealed that the critical behavior of the Ising and Potts models on complex networks strongly differs from the standard mean-field behavior on a regular lattice 11, 12, 13, 14, 15, 16, 17]. In the present section we analyze pair correlations in the equilibrium Ising model on an uncorrelated random complex network.

\section{A. The model}

We consider the ferromagnetic Ising model:

$$
\mathcal{H}=-J \sum_{\langle i j\rangle} S_{i} S_{j}-\sum_{i} H_{i} S_{i}
$$


where $S_{i}= \pm 1$, and $H_{i}$ is a local magnetic field at a vertex $i$. The sum is over all nearest neighboring vertices. The static pair-correlation function

$$
G_{i j} \equiv\left\langle S_{i} S_{j}\right\rangle-\left\langle S_{i}\right\rangle\left\langle S_{j}\right\rangle
$$

is related to the non-local magnetic susceptibility $\chi_{i j}$ :

$$
\chi_{i j}=\partial M_{i} / \partial h_{j}=\beta G_{i j},
$$

where $\beta=1 / T$ and $T$ is temperature, $M_{i} \equiv\left\langle S_{i}\right\rangle$. In this section $\langle\ldots\rangle$ means the statistical average with the Hamiltonian $\mathcal{H}$.

As a substrate, we use the standard model of an uncorrelated random network - the configuration model $[9]$. This is the maximally random graph with a given degree distribution or, as it is called in graph theory, a labelled random graph with a given degree sequence. It is important that the uncorrelated networks have a locally tree-like structure. That is, in the large network limit, there are no loops in a finite neighborhood of a vertex. These networks may be considered as the random Bethe lattices, which, by definition, have no boundary. One should note that in contrast, the Cayley tree contains boundary vertices which are dead ends [18].

\section{B. How to solve the Ising model on a tree-like graph}

Let us outline an effective approach to solution of cooperative models on tree-like networks, which we use for obtaining $\chi_{i j}$. This method was implemented for the Ising and Potts models on regular [18] and random [12, 19] Bethe lattices. Consider an arbitrary tree-like graph. Consider a spin $S_{i}$ on a vertex $i$ of this graph with $k_{i}$ adjacent spins $S_{j}, j=1,2, \ldots, k_{i}$. As any vertex in this graph, vertex $i$ may be treated as a root of a tree. In turn, vertices $j$ may be treated as roots of subtrees growing from the vertex $i$. In order to characterize the subtree with the root spin $S_{j}$ we introduce a quantity

$$
\begin{aligned}
g_{i j}\left(S_{i}\right)= & \sum_{\left\{S_{l}\right\}= \pm 1} \exp \left[\beta J \sum_{\langle n m\rangle} S_{n} S_{m}\right. \\
& \left.+\beta J S_{i} S_{j}+\beta \sum_{n} H_{n} S_{n}\right] .
\end{aligned}
$$

Here the indices $n$ and $m$ run only over spins that belong to the sub-tree, including $S_{j}$. The statistical sum in Eq. (13) is taken only over the spins on the subtree.

We introduce a quantity

$$
x_{i j} \equiv g_{i j}(-1) / g_{i j}(+1) .
$$

In such a way, for each vertex $i$ we can introduce $k_{i}$ parameters $x_{i j}, j=1,2, \ldots k_{i}$. It should be noted that $x_{i j} \neq x_{j i}$ because $x_{i j}$ and $x_{j i}$ characterize different subtrees. So, each edge is characterized by a pair: $x_{i j}$ and $x_{j i}$. In sum, the Ising model on an arbitrary tree-like graph is described by $2 L=\sum_{i} k_{i}$ parameters $x_{i j}$. Here, $L$ is the total number of edges of the graph.

Using Eqs. (13) and (14), the parameter $x_{i j}$ may be related to parameters $x_{j l}$ which characterize the edges (sub-trees) outgoing from the vertex $j$ as follows [18]:

$$
x_{i j}=y\left(H_{j}, \prod_{l=1}^{k_{j}-1} x_{j l}\right),
$$

where $l$ is the index of the first nearest neighbors of the vertex $j$. The vertex $i$ is excluded from the product over $l$. In other words, a vertex $l$ is a second neighbor of the vertex $i$ and the first one of the vertex $j$. The function $y(H, x)$ in Eq. (15) depends on a cooperative model. For the Ising model,

$$
y(H, x)=\frac{e^{(-J+H) \beta}+e^{(J-H) \beta} x}{e^{(J+H) \beta}+e^{(-J-H) \beta} x} .
$$

$2 L$ independent parameters $x_{i j}$ should be found by solution of the set of $2 L$ equations (15) at a given temperature $T$ and local magnetic fields $H_{i}$. For an arbitrary tree-like graph, these equations may be solved numerically, e.g., by use of the population dynamic algorithm, see Ref. 17] where this method has been applied to the Potts model on a tree-like graph.

Observable thermodynamic quantities of the Ising model may be written as functions of the parameters $x_{i j}$. For example, a magnetic moment $M_{i}$ is given by the following equation:

$$
M_{i}=\left(e^{2 \beta H_{i}}-\prod_{j=1}^{k_{i}} x_{i j}\right) /\left(e^{2 \beta H_{i}}+\prod_{j=1}^{k_{i}} x_{i j}\right) .
$$

Finally, for a random network, the resulting observables should be averaged over various configurations with appropriate statistical weights.

\section{Derivation of the pair correlation function}

Let us find a non-local susceptibility $\chi_{i j}$ for two arbitrary vertices $i$ and $j$ at the distance $l_{i j}=\ell$ from each other. On a tree-like graph there is the only shortest way connecting these vertices. It starts from $i$, then goes through vertices $i_{1}, i_{2}, \ldots, i_{\ell-1}$ and ends at $j$. Using Eqs. (17) and (15) we get

$$
\chi_{i j}=\frac{\partial M_{i}}{\partial x_{i i_{1}}} \frac{\partial x_{i i_{1}}}{\partial x_{i_{1} i_{2}}} \frac{\partial x_{i_{1} i_{2}}}{\partial x_{i_{2} i_{3}}} \ldots \frac{\partial x_{i_{l-2} i_{\ell-1}}}{\partial x_{i_{\ell-1} j}} \frac{\partial x_{i_{\ell-1} j}}{\partial H_{j}} .
$$

If we know $x_{i j}$ we can get $\chi_{i j}$.

First, for the purpose of comparison, we find a nonlocal susceptibility $\chi_{i j}$ of a regular Bethe lattice with a coordination number $k$.

In a uniform magnetic field $H_{1}=H_{2}=\ldots .=H$ all vertices and all edges in a regular Bethe lattice are 
equivalent. Therefore, the parameters $x_{i j}$ are equal, i.e. $x_{i j}=x$, and Eq. (15) is reduced to

$$
x=y\left(H, x^{k-1}\right) .
$$

This equation determines $x$ as a function of $T$ and $H$. From Eqs. (18) and (19) we get

$$
\chi(\ell)=\frac{1}{k} \frac{\partial M}{\partial x}\left[\frac{1}{(k-1)} \frac{\partial y\left(H, x^{k-1}\right)}{\partial x}\right]^{\ell-1} \frac{\partial y\left(H, x^{k-1}\right)}{\partial H},
$$

where in accordance with Eq. (17) we have $M=\left(e^{2 \beta H}-\right.$ $\left.x^{k}\right) /\left(e^{2 \beta H}+x^{k}\right)$. At zero magnetic field $H=0$, Eq. (20) takes a form:

$$
\chi(\ell)=\frac{4 \beta x^{k}}{\left(1+x^{k}\right)^{2}}\left[\frac{2 x^{k-2} \sinh (2 J \beta)}{\left(e^{J \beta}+e^{-J \beta} x^{k-1}\right)^{2}}\right]^{\ell} .
$$

This equation is valid at all temperatures.

In the paramagnetic state, i.e. at temperatures $T$ above the critical temperature $T_{c}=2 J / \ln [k /(k-2)]$ of the ferromagnetic phase transition, the self-consistent equation (19) has the only solution $x=1$, and we get

$$
\chi(\ell)=\beta G(l)=\beta \tanh ^{\ell}(J \beta)
$$

in agreement with the result obtained in Ref. 20 for a Cayley tree in the framework of another method. Furthermore, using the fact that $x_{i j}=1$ at $T \geq T_{c}$, one can show that in the paramagnetic phase, $\chi(\ell)$ of the Ising model on an arbitrary tree-like graph is the same as that for an arbitrary Cayley tree, e.g., for a spin chain (see below). Recall that there is no phase transition in spin models on a Cayley tree due to the presence of boundary spins. The number of these spins is of the order of the total number of spins on a given Cayley tree.

In the ferromagnetic phase, i.e. at $T<T_{c}$, or at $H \neq 0$ the parameter $x$ is smaller than 1 . Note that in accordance with Eqs. (21) and (22) the non-local susceptibility $\chi(\ell)$ of a regular Bethe lattice is finite at all temperatures and magnetic fields.

Now let us consider an uncorrelated random network. We calculate a value of $\chi_{i j}\left(\ell_{i j}=\ell\right)$ averaged over the ensemble of uncorrelated random graphs with a given degree distribution function $P(k)$ :

$$
\begin{gathered}
\chi(\ell) \equiv \overline{\chi_{i j}\left(\ell_{i j}=\ell\right)}=\sum_{k_{i}} \sum_{k_{1}} \ldots \sum_{k_{l-1}} \sum_{k_{j}} \chi_{i j} \\
\times\left[\frac{P\left(k_{j}\right) k_{j}}{z_{1}}\left\{\prod_{n=1}^{\ell-1} \frac{P\left(k_{n}\right) k_{n}\left(k_{n}-1\right)}{z_{2}}\right\} \frac{P\left(k_{i}\right) k_{i}}{z_{1}}\right] .
\end{gathered}
$$

Here, the quantity in the square brackets is the probability that a vertex $i$ with degree $k_{i}$ is connected with a vertex $j$ having degree $k_{j}$ by a path that goes through vertices with degrees $k_{1}, k_{2}, \ldots k_{\ell-1}$.

In the paramagnetic phase at $T \geq T_{c}=$ $2 J / \ln \left[\left\langle k^{2}\right\rangle /\left(\left\langle k^{2}\right\rangle-2\langle k\rangle\right)\right]$ and $H=0$ we have $x_{i j}=1$, and Eq. (23) leads to the Eq. (22). Therefore, in zero magnetic field both on regular and random Bethe lattices the non-local susceptibility and $\chi_{i j}$ have the same temperature dependence determined by Eq. (22).

At a magnetic field and in the ferromagnetic phase an approximate expression for the function $\chi(\ell)$ may be obtained in the framework of the approach proposed in [12]. We introduce $x_{i j}=\exp \left(-h_{i j}\right)$. Here, the quantities $h_{i j}$ are positive and independent random parameters. We use the following ansatz,

$$
\sum_{j=1}^{k} h_{i j} \approx k h+\mathcal{O}\left(k^{1 / 2}\right)
$$

where $h \equiv \overline{h_{i j}}$ is the average value of the parameter. Applying the ansatz (24) to Eq. (15) yields

$$
h=-\frac{1}{\bar{k}} \sum_{k} P(k) k \ln y\left(H, e^{-(k-1) h}\right)
$$

which determines $h$ as a function of $T$ and $H$. In fact, the parameter $h$ plays the role of the order parameter. At $H=0$, we get $h=0$ above $T_{c}$ and non-zero below $T_{c}$.

With this ansatz,

$$
\chi(\ell)=z_{1}^{2} z_{2}^{-(\ell-1)} A B^{\ell-1} C,
$$

where

$$
\begin{gathered}
A=-\sum_{q} q P(q) \frac{2 e^{-q h}}{\left(e^{H \beta}+e^{-H \beta-q h}\right)^{2}} \\
B=\sum_{q} q(q-1) P(q) \frac{2 e^{-(q-2) h} \sinh (2 J \beta)}{\left(e^{(J+H) \beta}+e^{-(J+H) \beta-(q-1) h}\right)^{2}} \\
C=-\sum_{q} q P(q) \frac{4 e^{-(q-1) h} \sinh (2 J \beta)}{\left(e^{(J+H) \beta}+e^{-(J+H) \beta-(q-1) h}\right)^{2}}
\end{gathered}
$$

The quantities $A, B$ and $C$ are finite (independent of $N$ ) for an arbitrary degree distribution $P(k)$. The finiteness of the sums in Eqs. (27)-29) is ensured by the exponential multiplier $e^{-q h}$.

Result (26) is in agreement with the conclusions of the preceding section [compare relations (26) and (8)]. Note that the conclusion that $\chi(\ell \geq 2) \propto G(\ell \geq 2) \rightarrow 0$ as $N \rightarrow \infty$ if the second moment of the degree distribution diverges, does not depend on the fact that we used the approximation (24). Indeed, substituting Eq. (18) into Eq. (23), one can prove that all the sums over degrees $k_{n}$ converge both in the ordered state and at $H \neq 0$. Therefore, we get $\chi(\ell) \propto z_{2}^{-(\ell-1)}$ in agreement with Eq. (26).

\section{DISCUSSION AND CONCLUSIONS}

In the framework of a phenomenological approach and by using the Ising model we have studied time and static 
pair correlations $\left\langle x_{i}\left(t_{1}\right) x_{j}\left(t_{2}\right)\right\rangle$ between interacting objects defined on a random uncorrelated complex network. We have demonstrated that if a complex network has a divergent number of the second neighbors, then the pair correlations between second and more distant neighbors are strongly suppressed in large networks. In fact, the correlations tend to zero in the limit $N \rightarrow \infty$. One should note that a particular form of the characteristic $z_{2}(N)$ strongly depends on a network model. It is important that the size dependence of the pair correlation function is determined by the factor $1 / z_{2}^{\ell-1}(N)$.

The effect of weakening of the pair correlations is well known to people leaving in large towns and forming a large network of acquaintances. Close relationships are only being kept between close relatives inside family, close friends or between people working in the same office. These people are our nearest neighbors. Our "second" and more distant nearest neighbors - people living on the next floor or working in a neighboring office usually weakly influence our personal and social life. This is contrary to a small village where strong correlations are present between almost all inhabitants.

Our analysis may be generalized to correlated networks. Many natural networks exhibit correlations between degrees of adjacent vertices, see, for example, Ref. 21]. Note that in large uncorrelated networks, the divergence of the mean number $z_{\ell}$ of the $\ell$-th nearest neighbor can occur only simultaneously at all $\ell \geq 2$. In contrast, in large correlated networks, it is possible in principle that, say, $z_{2}, z_{3}$ is finite and only $z_{\ell \geq 4}$ diverges. In this example, pair correlations are observable between the first, second and third nearest neighbors and vanish starting from the fourth nearest neighbors.

We restricted ourselves to pair correlations. One should emphasize that pair correlations in cooperative systems on networks, unlike lattices, never show critical behavior. Even at the critical point of an interacting system on a network, we observe an exponentially rapid decay of pair correlations. In contrast, volume correlations in interacting systems on networks demonstrate a critical feature. Let us explain this point. The distribution of the full response of a system to a small local field is $P(\varepsilon)=\sum_{i} \delta\left(\sum_{j} \chi_{i j}-\varepsilon\right)$, where $\delta(\varepsilon)$ is the delta-function. This distribution is a rapidly decreasing function both below and above a phase transition on a network. On the other hand, at the critical point, $P(\varepsilon)$ is power-law, that is, "critical" 10, 22].

One can conclude that a network structure of an interacting system strongly influences the dependence of pair correlations on the distance between interacting objects. So, investigations of the correlations may be a useful method to understand the network topology. If an interacting system is defined on a network with a divergent number $z_{n}$ of $n$-th nearest neighbors of a vertex, then dynamic and static pair correlation are strongly suppressed at distances $n$ and greater.

This work was partially supported by projects POCTI: FAT/46241/2002, MAT/46176/2002, FIS/61665/2004, and BIA-BCM $/ 62662 / 2004$. S.N.D. and J.F.F.M. were also supported by project DYSONET-NEST/012911. Authors thank A.N. Samukhin for useful discussions.
[1] S.H. Strogatz, Nature 401, 268 (2001).

[2] R. Albert and A.-L. Barabási, Rev. Mod. Phys. 74, 47 (2002).

[3] S.N. Dorogovtsev and J.F.F. Mendes, Adv. Phys. 51, 1079 (2002).

[4] M.E.J. Newman, SIAM Review 45, 167 (2003).

[5] D.J. Watts, Small Worlds: The Dynamics of Networks between Order and Randomness (Princeton University Press, Princeton, NJ, 1999).

[6] S.N. Dorogovtsev and J.F.F. Mendes, Evolution of Networks: From Biological Nets to the Internet and $W W W$ (Oxford, University Press, 2003).

[7] R. Pastor-Satorras and A. Vespignani, Evolution and Structure of the Internet: A Statistical Physics Approach (Cambridge University Press, Cambridge, 2004).

[8] R. Albert, H. Jeong, and A.-L. Barabási, Nature 401, 130 (1999).

[9] A. Bekessy, P. Bekessy, and J. Komlos, Stud. Sci. Math. Hungar. 7, 343 (1972); E.A. Bender and E.R. Canfield, J. Combinatorial Theory A 24, 296 (1978); B. Bollobás, Eur. J. Comb. 1, 311 (1980); N.C. Wormald, J. Combinatorial Theory B 31, 156,168 (1981).

[10] M. E. J. Newman, S. H. Strogatz, and D. J. Watts, Phys. Rev. E 64, 026118 (2001).

[11] A. Aleksiejuk, J.A. Holyst, and D. Stauffer, Physica A
310, 260 (2002)

[12] S.N. Dorogovtsev, A.V. Goltsev and J.F.F. Mendes, Phys. Rev. E 66, 016104 (2002).

[13] M. Leone, A. Vázquez, A. Vespignani, and R. Zecchina, Eur. Phys. J. B 28, 191 (2002).

[14] G. Bianconi, Phys. Lett. A 303, 166 (2002).

[15] R. Cohen, K. Erez, D. ben-Avraham, and S. Havlin, Phys. Rev. Lett. 85, 4626 (2000).

[16] D.-S. Lee, K.-I. Goh, B. Kahng, and D. Kim, Nucl. Phys. B 696, 351 (2004).

[17] G.C.M.A. Ehrhardt and M. Marsili, cond-mat/0411226

[18] R.J. Baxter, Exactly Solved Models in Statistical Mechanics (Academic Press, London, 1982).

[19] S.N. Dorogovtsev, A.V. Goltsev, and J.F.F. Mendes, Eur. Phys. J. B 38, 177 (2004).

[20] H. Falk, Phys. Rev. B 12, 5184 (1975).

[21] M.E.J. Newman, Phys. Rev. Lett. 89, 208701 (2002); M.E.J. Newman, Phys. Rev. E 67, 026126 (2003); S. Maslov and K. Sneppen, Science 296, 910 (2002); E. Ravasz, A.L. Somera, D.A. Mongru, Z.N. Oltvai, and A.-L. Barabási, Science 297, 1551 (2003).

[22] M. Bauer, S. Coulomb, and S. N. Dorogovtsev, Phys. Rev. Lett. 94, 200602 (2005). 